\title{
ImPaCt Of Multipath Clustering on The Performance of MiMO Systems
}

\author{
Guangze Zhao, Sergey Loyka \\ School of Information Technology and Engineering (SITE) \\ University of Ottawa, 161 Louis Pasteur, Ottawa, Ontario, K1N 6N5, Canada
}

\begin{abstract}
Fading correlation has a profound impact on MIMO system performance. Salz-Winters model is a popular tool to study this effect. However, it is limited to one cluster only. Measurements indicate that multipath often arrives in several clusters. We extend the Salz-Winters model to the case of multicluster channels and study it in detail. Closed-form expression for correlations are derived and applied to MIMO capacity/diversity gain analysis. The maximum gain/capacity are achieved provided that a minimum element spacing (derived in the paper) is respected. It is shown that the correlation has an oscillatory behavior as antenna spacing increases; the envelope of correlation is determined by a single cluster angular spread while the oscillations within the envelope are determined by the intercluster angular spread. It is demonstrated that the correlation depends significantly on the power distribution among the clusters. In the case of 2 widely-separated clusters it is possible to orient the antenna array in such a way that the correlation is minimized and, hence, the capacity/gain are maximized. We study this optimization problem and derive the optimum array orientation. Overall, the paper presents a new insight on correlation properties of multipath clustered channels, and on the MIMO system performance over such channels.
\end{abstract}

Index Terms-Wireless propagation channel, fading correlation, MIMO system

\section{INTRODUCTION}

Radio propagation channel plays a crucial role in the performance analysis of wireless systems. Multipath effects result in a fading channel, which may degrade the system performance substantially. Multi-antenna systems are used to take advantage of multipath propagation, but they are very sensitive to fading correlation from one antenna to another. Hence, accurate analysis and simulations of the correlation are required. One of the most popular correlation models is the Salz-Winters model [1]. It allows to predict the correlation (with reasonable degree of accuracy) assuming that all the multipath components arrive within given angular spread around the average angle of arrival. By varying the angular spread and the average angle of arrival, many practical scenarios can be modeled and measured data can be approximated as well. However, a significant drawback of the Salz-Winters model, as well as its many extensions and other similar models is that only one cluster is allowed. Measurements indicate that multipath components are frequently concentrated within two or more clusters [6-8]. Hence, the Salz-Winters model cannot be applied in this case. It was further extended to a multi-cluster case in $[10,11]$. However, only preliminary results were reported; no detailed study has been undertaken.

In this paper, we use the extended Salz-Winters model to account for more than one cluster and study in details the effect of clustering. Multipath components arriving in different clusters are assumed to be uncorrelated (this assumption is justified by the physical mechanism of clustering) and uniformly angular-distributed within the corresponding clusters. A case of two clusters is studied in details, including both symmetrical and asymmetrical location of the clusters, and equal/unequal angular spreads and power distributions. The correlation coefficient has an oscillatory behavior with respect to the antenna spacing, which is in good agreement with [11]. As a cluster moves away from the array broadside direction, its contribution to the total correlation decreases (inverse cosine law). In the case of identical symmetricallylocated clusters, the envelope of correlation is determined by a single cluster angular spread while the oscillations within the envelope are determined by the inter-cluster angular spread (for both clusters). If the clusters are identical and located asymmetrically (i.e., broadside-endfire), the impact of the endfire cluster is, in many cases, much smaller and can be neglected for proportional power allocation (see section III). Hence, one-cluster model can be used, which simplifies the analysis substantially.

An extension to the case of n-cluster scenario is straightforward. The major analytical techniques developed above are applicable in this general case as well. The correlation is presented as a series of terms, each term representing a cluster. This simplifies the comparison of individual contributions of the clusters to the total correlation.

While studying correlation versus element spacing behavior, we derive a closed-form expression for the minimum element spacing that provides low correlation and, hence, large capacity or diversity gain. Closed-from analytical expressions for the correlation coefficient, including Bessel series expansions and compact approximate expressions, are derived for many scenarios, and validated through Monte-Carlo simulations.

Using the correlation models developed above and the technique presented in $[2,3]$, we study diversity combining gain and MIMO capacity. In particular, we demonstrate that the maximum gain/capacity is achieved provided that the minimum element spacing derived above is respected. When two cluster are widely separated (i.e., the angular separation is larger than the cluster widths), it is possible to orient the antenna array in such a way that the correlation is minimized and, hence, the capacity/gain are maximized.

The results presented agree well with those published in the literature earlier and extend them to the case of multi-cluster channels. Approximate analytical solutions presented allow for deeper insight and understanding of the clustering phenomenon.

\section{SALZ-WINTERS MODEL}

In this model, all the multipath component are assumed to arrive to the $\mathrm{Rx}$ array within $\pm \Delta / 2$ of the mean angle of arrival (AOA) $\varphi$ (see fig. 1) in the horizontal plane (i.e., this is 
a 2-D model). The AOA probability density function is assumed to be uniform,

$$
f_{\theta}(\theta)=\left\{\begin{array}{c}
1 / \Delta,-\Delta / 2+\varphi \leq \theta \leq \Delta / 2+\varphi \\
0, \text { otherwise }
\end{array}\right.
$$

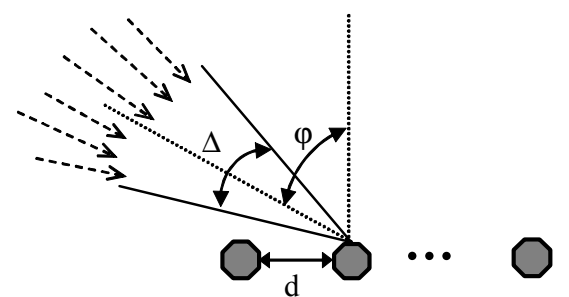

Figure 1. Incoming multipath signals arrive to the linear antenna array within $\pm \Delta / 2$ of mean angle $\varphi$

In a general case, the normalized correlation between two array elements of a uniform linear array (ULA) can be expressed as

$$
R=\int_{-\pi}^{\pi} f_{\theta}(\theta) \exp [j z \sin \theta] d \theta
$$

where $z=2 \pi d / \lambda, d$ is the element spacing, $\lambda$ is the wavelength, and $j$ is the imaginary unit. Without loss of generality, we further assume that $\lambda=1$. Substituting (1) in (2), one obtains the Bessel series expansion for R [1]. For $\Delta=2 \pi$, it reduces to the classical Jakes expression [4], $R=J_{0}[z]$. The Bessel series expansion, as detailed analysis shows, converges very slowly and, consequently, a large number of terms must be used in order to estimate $R$ accurately for small $\Delta$ (a few degrees) [2]. The computational efficiency of this procedure is very low, especially when matrix computations are involved, as is the case for MIMO systems.

A simple but still accurate approximation of the Bessel series expansion for small $\Delta<<1$ can be derived using $\sin \beta \approx \beta$ (valid for small $\beta$ ), and performing integration in (2):

$$
R \approx \operatorname{sinc}(\Delta d \cos \varphi) e^{j 2 \pi d \sin \varphi}
$$

where $\operatorname{sinc}(x)=\sin (\pi x) /(\pi x)$. The smaller $\Delta$, the better the accuracy is. Thus, this approximation works exactly where it is needed. The upper bound of its validity is approximately $\pi / 2$. Hence, one may use the Jakes expression for large values of $\Delta$ and (3) for small ones. For $\varphi=0$, (3) reduces to $R \approx \operatorname{sinc}(\Delta d)$, which is the same as [2, eq. 3]. This immediately explains eq. 13 in [2] (which was actually a conjecture): using an "effective" spacing $d^{\prime}=d \cos \varphi$ (the spacing seen at angle $\varphi$ ), the effect of the cluster average AOA is to decrease the "effective" element spacing, which is identical to a similar effect in the antenna array theory [9] (the effective array aperture is decreased in the same way). Hence, the minimum correlation and the best performance (MIMO capacity or diversity gain) are achieved when $\varphi=0$. It is

\footnotetext{
${ }^{1}$ After the paper has been submitted, it was brought to our attention that similar results also appear in $[10,11]$.
}

remarkable that such a simple model is able to explain this well-known effect.

It should be noted that the assumption of uniform angular pdf does not limit significantly the accuracy of the model. As detailed analysis shows, the non-uniform pdf models (i.e., Laplacian, truncated Gaussian etc.) produce roughly the same results (in term of MIMO capacity and diversity gain). Intuitively, it can be explained by two factors: (i) correlation is an integral of the angular pdf (see (2)); hence, all the local variations in $f_{\beta}(\beta)$ are smoothed out during the integration and do not affect significantly the final result (provided that the angular spread is the same for all the distributions), i.e. this is a second-order effect; (ii) system performance is not affected significantly when the correlation is less than 0.5 , where the deference between uniform and non-uniform distributions is the largest. Similar conclusion has also been presented in [10].

\section{TWO-CLUSTER MODEL}

Measurements indicated that multipath arrives frequently in more than one cluster [6-8]. Hence, generalizing Salz-Winters model, we assume the angular pdf of the form,

$$
p(\varphi)=\left\{\begin{array}{l}
\frac{1}{\Delta_{1}+\Delta_{2}},\left\{\begin{array}{l}
-\Delta_{1} / 2+\varphi_{1} \leq \varphi \leq \Delta_{1} / 2+\varphi_{1}, \\
-\Delta_{2} / 2+\varphi_{2} \leq \varphi \leq \Delta_{2} / 2+\varphi_{2}
\end{array}\right. \\
0, \text { elsewhere }
\end{array}\right.
$$

The scenario is illustrated in Fig. 2. Note that the total power in each cluster depends on the angular spread - we call it "proportional power allocation".

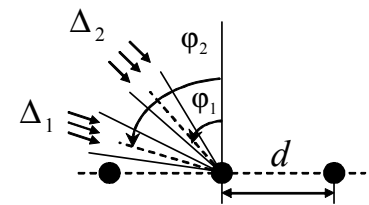

Figure 2. Incoming multipath is concentrated in two clusters.

Substituting (4) in (2), we obtain,

$$
\begin{aligned}
& R=R_{x x}+j R_{x y} \\
& R_{x x}=J_{0}(z)+2 \sum_{m=1}^{\infty} J_{2 m}(z)\left(R_{1 m}^{x x}+R_{2 m}^{x x}\right) \\
& R_{x y}=2 \sum_{m=0}^{\infty} J_{2 m+1}(z)\left(R_{1 m}^{x y}+R_{2 m}^{x y}\right)
\end{aligned}
$$

where $J_{m}$ is the first-kind Bessel function of order m, and

$$
\begin{aligned}
R_{i m}^{x x} & =\frac{\cos \left(2 m \varphi_{i}\right) \sin \left(m \Delta_{i}\right)}{m\left(\Delta_{1}+\Delta_{2}\right)}, \\
R_{i m}^{x y} & =2 \frac{\sin \left[(2 m+1) \varphi_{i}\right] \sin \frac{(2 m+1) \Delta_{i}}{2}}{(2 m+1)\left(\Delta_{1}+\Delta_{2}\right)}
\end{aligned}
$$

It should be noted that (5) reduces to the Salz-Winters model when $\varphi_{2}=\varphi_{1}+\left(\Delta_{1}+\Delta_{2}\right) / 2$, i.e. when two clusters merge into one bigger cluster, as it should be.

In order to get some insight into correlation behavior of a two-cluster channel, we now study some special cases.

A. Two symmetric clusters

In this case, $\Delta_{1}=\Delta_{2}=\Delta$ and $\varphi_{1}=-\varphi_{2}=\varphi$. (5) reduces to 


$$
R=J_{0}(z)+2 \sum_{m=1}^{\infty} J_{2 m}(z) \frac{\cos (2 m \varphi) \sin (m \Delta)}{m \Delta}
$$

The correlation is real and it is the same as the real part of the single-cluster correlation. Hence, the effect of the second cluster (which is symmetric to the first one w.r.t. the array broadside) is to cancel the imaginary part of the correlation. The real part is not changed simply because both clusters contribute equally to the real part (due to the symmetry) but each cluster has twice less power than the single cluster.

For small $\Delta$ convergence of (6) is very slow (i.e. many terms should be kept to get good accuracy). A better way is to solve (2) directly using the same approximation that leads to (3),

$$
R \approx \cos (z \sin \varphi) \operatorname{sinc}(\Delta d \cos \varphi)
$$

Note that (7) can be obtained using (3): $R=\left(R_{3}+R_{3}^{*}\right) / 2$, where $R_{3}$ is given by (3). This supports our interpretation above. While the real parts of (3) and (7) are the same, their magnitudes are very different, as Fig. 3 illustrates. In fact, the single-cluster magnitude correlation is the envelope of the twocluster correlation and the cosine function in (7) results in oscillation in the latter case.

$R=0$ when either $\cos (z \sin \varphi)=0$ or $\operatorname{sinc}(\Delta d \cos \varphi)=0$. The element spacing at these points is

$$
d_{0}=\left\{\begin{array}{l}
\frac{m_{1}}{4 \sin \varphi}, m_{1}=1,3,5, \cdots,(2 k-1), \cdots \\
\frac{m_{2}}{\Delta \cos \varphi}, m_{2}=1,2,3, \cdots, k, \cdots
\end{array}\right.
$$

First-type zeros are due to the cosine and second-type zeros are due to the sinc. The spacing between first-type zeros is $\Delta d_{01}=1 /(2 \sin \varphi)$ and it does not depend on the angular spread $\Delta$; the zero spacing of the second- type zeros is $\Delta d_{02}=1 /(\Delta \cos \varphi)$ and its does depend on the angular spread - the larger the spread the smaller the spacing. $\cos \varphi$ law mentioned above is also obvious. The second- type zeros are the same as for the single cluster model (see eq. 3). The minimum spacing for the first-type zeros is $d_{01, \min }=1 /(4 \sin \varphi)$ and the minimum spacing for the secondtype zeros is $d_{02, \min }=1 /(\Delta \cos \varphi)$. Fig. 3 validates (6)-(8) using Monte-Carlo simulations. Note that, in this case, $d_{01, \min }=0.5 \lambda, \Delta d_{01}=\lambda$ and $d_{02 \text {, min }}=6.6 \lambda$, i.e. the $2^{\text {nd }}$ type zero spacing is much large than that of the $1^{\text {st }}$ type.

Good system performance (capacity, diversity gain etc.) requires for low (ideally - zero) correlation. Normally, this is accomplished by setting d large enough. However, as (8) demonstrates, in a two-cluster scenario the spacing $\mathrm{d}$ can also be set, when possible, at zero locations. For small $\Delta$ and when $\varphi$ is not too close to $0, d_{01, \min }<d_{02 \text {,min }}$, and, hence, the twocluster effect of oscillation can be beneficially used to achieve low correlation at smaller $d=d_{01, \min }=1 /(4 \sin \varphi) \quad$ (as compared to the single-cluster case). Note however that, in this case, zero spacing correlation will be achieved for all the pairs spaced apart by $m_{1} d$; all the pair spaced by even number of $d$

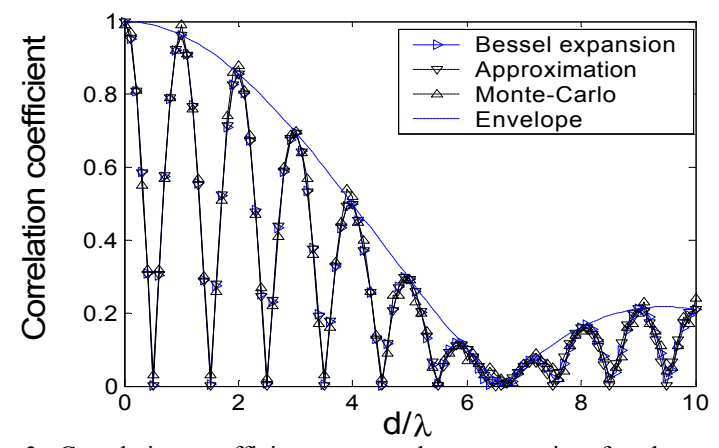

Fig. 3. Correlation coefficient versus element spacing for the case of two symmetric clusters. $\Delta=10^{0}, \varphi=30^{\circ}$

may be correlated. Hence, the performance is guaranteed to be the best only in the case of 2-element Rx antenna. This does not happen when $d=d_{02 \text {,min }}$ : all the pair wise correlations are zero in this case and the performance is the best for any $n$.

In order to accomplish correct spacing setting, $\varphi$ and/or $\Delta$ must be known quite accurately or zero spacing must be measured, which may not be feasible in practice. In this case, $d$ should be set large enough - the same as for the single-cluster case.

It is instructive to consider the limiting case of $\Delta \rightarrow 0$. In this case, the angular pdf $f_{\theta}(\theta)=1 / 2(\delta(\theta-\varphi)+\delta(\theta+\varphi))$, where $\delta$ is the Dirac delta-function, and

$$
R=\cos (2 \pi d \sin \varphi)
$$

i.e. the correlation $\mathrm{R}$ is a periodic function in $\mathrm{d}$ and it never decays to zero (as is the case for $\Delta \neq 0$ ). In this case, large $\mathrm{d}$ does not guarantee low correlation unless it is set equal to the zero spacing.

Measurements results reported in [5] indicated that the correlation did not decrease with increasing the element spacing in certain scenarios. Rather, it was oscillatory in nature. The two-cluster model above gives a simple explanation for this phenomenon.

\section{B. Two asymmetric clusters}

In this case, we assume that the angular spreads are small, $\Delta_{1}, \Delta_{2}<<1, \Delta_{1} \neq \Delta_{2}$, and one cluster is located at the broadside direction, $\varphi_{1}=0$. Substituting (4) in (2), one obtains

$$
R \approx w_{1} R_{1}+w_{2} R_{2}
$$

where $R_{1}$ and $R_{2}$ are single-cluster correlations given by (3),

$$
\begin{gathered}
R_{1}=\operatorname{sinc}\left(\Delta_{1} d\right), R_{2}=\operatorname{sinc}\left(\Delta_{2} d \cos \varphi_{2}\right) e^{j 2 \pi d \sin \varphi_{2}}, \\
w_{1}=\Delta_{1} /\left(\Delta_{1}+\Delta_{2}\right), w_{2}=\Delta_{2} /\left(\Delta_{1}+\Delta_{2}\right)=1-w_{1} .
\end{gathered}
$$

Note that single-cluster correlations are weighted by $w_{1}$ and $w_{2}$. This is due to the implicit assumption in (4) that the angular power density is the same for both clusters, i.e. the actual power within given cluster depends on its spread. 
Correlation in (10) also has oscillatory behavior due to constructive and distractive addition of the two terms.

The maxima and minima of the correlation magnitude occur at approximately $\cos \left(z \sin \varphi_{2}\right)= \pm 1$, which leads to the following

$$
\begin{aligned}
& |R|_{\text {max }}=R\left(d_{\text {max }}\right), d_{\text {max }}=k / \sin \varphi_{2} \\
& |R|_{\text {min }}=R\left(d_{\text {min }}\right), d_{\text {min }}=(k+1 / 2) / \sin \varphi_{2}
\end{aligned}
$$

where $k=0,1,2,3, \ldots$. Fig. 4 illustrates this effect. Note that, in this case, $d_{\max }=2 k \lambda$ and $d_{\min }=(2 k+1) \lambda$, in full agreement with (11). One can also see that the contribution of the $2^{\text {nd }}$ cluster becomes more pronounced as $\Delta_{2}$ increases (again, in full agreement with (10)). Comparison of Fig. 3 and 4 illustrates the difference between symmetric and asymmetric cluster cases.

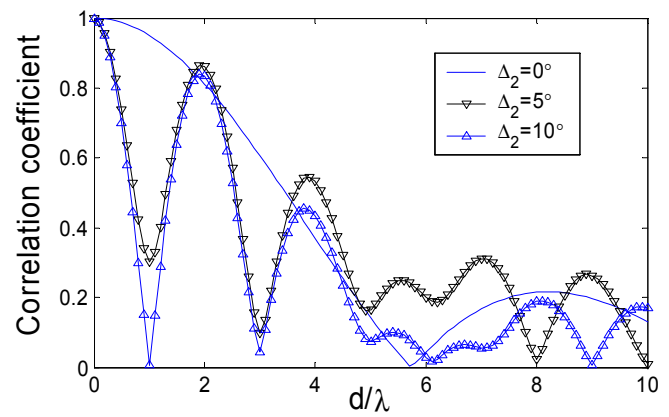

Fig. 4 Correlation for two asymmetric clusters with small angular spreads. $\Delta_{1}=10^{0}, \varphi_{1}=0^{0}, \varphi_{2}=30^{0}$

As in the symmetric cluster case, setting the element spacing equal to $d_{\min }$ will result in zero pair-wise correlations for the odd-spaced pairs and the correlation of evenly-spaced pairs will not necessarily be low.

Considering $R(d), R_{1}(d)$ and $R_{2}(d)$ in (10), one may observe the following. The first zero of $R_{1}(d)$ is $d_{01}=1 / \Delta_{1}$ and $R_{1}$ is small for $d \geq d_{01}$. Hence, we say then that the rate of decay of $R_{1}(d)$ is $\Delta_{1}$. Similarly, $d_{02}=1 /\left(\Delta_{2} \cos \varphi\right)$ and the rate of decay of $R_{2}(d)$ is $\Delta_{2} \cos \varphi$. When $\Delta_{1}>\Delta_{2} \cos \varphi$, the first term in (10) is dominant for $d \in\left[0, d_{f}\right]$ and the $2^{\text {nd }}$ term is dominant for $d \in\left[d_{f}, \infty\right]$, where $\left|R_{1}\left(d_{f}\right)\right|=\left|R_{2}\left(d_{f}\right)\right|$. Approximately, $d_{f} \approx 1 /\left(\Delta_{2} \cos \varphi_{2}\right)$. $R(d)$ decays quickly on the $1^{\text {st }}$ interval and more slowly on the $2^{\text {nd }}$. In a sense, $R_{2}(d)$ provides a "floor" for $R(d)$ when $d>d_{f}$. Hence, when the spacing is small, neglecting the $2^{\text {nd }}$ cluster does change the correlation significantly.

When $\Delta_{1}$ is very large, $\Delta_{1}>\Delta_{2}$, the $2^{\text {nd }}$ term can be neglected for all values of $d$. In this sense, the correlation of a channel with one large cluster is less sensitive to the presence of other clusters. In particular, when $\Delta_{1} \geq \pi$, one may assume that $R \approx J_{0}(2 \pi d)$ regardless of whether there are other clusters.

\section{C. $\quad$ Two fixed-power clusters}

In many cases, the power of multipath components arriving in a cluster is fixed and does not depend on the angular spread of that cluster. In this case, the angular pdf for a two-cluster model is

$$
p(\varphi)=\left\{\begin{array}{cc}
\alpha / \Delta_{1}, & -\Delta_{1} / 2+\varphi_{1} \leq \varphi \leq \Delta_{1} / 2+\varphi_{1}, \\
(1-\alpha) / \Delta_{2}, & -\Delta_{2} / 2+\varphi_{2} \leq \varphi \leq \Delta_{2} / 2+\varphi_{2} \\
0, \text { elsewhere }
\end{array}\right.
$$

where constant $\alpha$ is the probability that the AOA falls within cluster 1 and it is proportional to the power content of cluster 1. $\alpha /(1-\alpha)$ is the power ratio of the two clusters. Since it does not depend on the angle spread, we call it "fixed power allocation".

For small $\Delta_{1}, \Delta_{2}<<1, \varphi_{1}=0$ and $\varphi_{2}$ being not too close to $90^{\circ}$, we obtain

$$
R \approx \alpha R_{1}+(1-\alpha) R_{2}
$$

where $\quad R_{1}=\operatorname{sinc}\left(\Delta_{1} d\right), \quad R_{2}=\operatorname{sinc}\left(\Delta_{2} d \cos \varphi_{2}\right) e^{j 2 \pi d \sin \varphi_{2}}$, which is identical to (10) with $w_{1}=\alpha$ and $w_{2}=1-\alpha$, as it intuitively should be. The different weights, which do not depend anymore on the angular spreads, have however a profound effect on the correlation behavior with the angular spread. For example, when one angular spread equals to zero, this cluster gives the dominant contribution to the total correlation, as opposed to the proportional power allocation of section $\mathrm{B}$, where this cluster did not contribute anything. Consider, for example, the case of $\alpha=1 / 2$. In this case, $R=\left(\operatorname{sinc}\left(\Delta_{1} d\right)+\operatorname{sinc}\left(\Delta_{2} d \cos \varphi_{2}\right) e^{j 2 \pi d \sin \varphi_{2}}\right) / 2$ and the $2^{\text {nd }}$ cluster provides the same contribution to the correlation as the $1^{\text {st }}$ one when $\Delta_{1}=\Delta_{2} \cos \varphi_{2}$. Clearly, the larger $\varphi_{2}$, the larger $\Delta_{2}$ is required for equal contribution. When $\Delta_{1}>\Delta_{2} \cos \varphi_{2}$, the $2^{\text {nd }}$ cluster gives dominant contribution. Hence, as a general tendency, the clusters located closely to the endfire direction provide small contribution only when they have larger angular spread as compared to the clusters located closely to the broadside directions. In the limiting case of $\Delta_{1}>\Delta_{2}$, the $1^{\text {st }}$ cluster can be totally ignored. For example, when $\Delta_{1} \geq 1 / d$ and $\Delta_{2}=0, R \approx e^{j 2 \pi d \sin \varphi_{2}} / 2$ (i.e., $|R| \approx 1 / 2$ ) regardless of $\Delta_{1}$. This is in sharp contrast to case B (recall that in Case B the $2^{\text {nd }}$ cluster can be totally ignored and one would obtain $\left.|R|=\left|\operatorname{sinc}\left(\Delta_{1} d\right)\right|<<1\right)$. Hence, power allocation among clusters has a dominant effect on the correlation. In general, the larger the power, the larger the contribution of that cluster to the correlation.

Fig. 5 illustrates this point. Comparing it with Fig. 4, one can clearly see the difference. It is instructive to consider the case of $\Delta_{2}=0$. In this case, the correlation periodically oscillates and its peak value never decreases below $1 / 2$, regardless of $d$. This is in sharp contrast with case $B$, where the correlation peaks can be decreased to any small value by sufficiently increasing $\mathrm{d}$. 


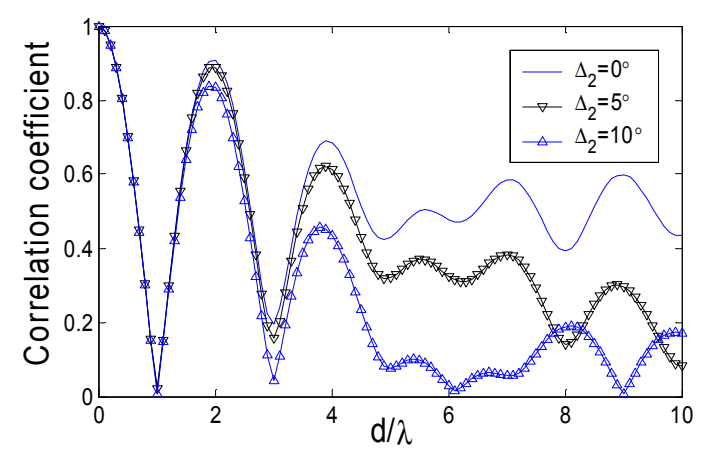

Fig. 5 Correlation for two asymmetric clusters with the same power.

\section{UNIFORM CIRCULAR ARRAY}

All the results obtained so far apply to the uniform linear array. In this section, we consider a uniform circular array (UCA) and reformulate the single-cluster model for this type of array. As one will see, the results can be sometime dramatically different.

The major difference between ULA and UCA is that the latter is not shift-invariant, i.e. the correlation between elements $\mathrm{i}$ and $\mathrm{j}$ depends not only on $|i-j|$ (i.e., the spacing) but also on the location on this particular pair since the broadside direction varies as we move the pair along the circle. Hence, the correlation will depend not only on the element spacing, but also on the location of these elements on the circle.

In a single-cluster channel, the correlation coefficient of elements $i$ and $k$ can be evaluated using (1) and (2),

$$
R_{i k}=\frac{1}{\Delta} \int_{\varphi-\varphi_{i k}-\Delta / 2}^{\varphi-\varphi_{i k}+\Delta / 2} e^{j 2 \pi d_{i k} \sin \beta} d \beta
$$

where $\varphi_{i}$ is the azimuth of i-th element, $\varphi_{i}=(2 \pi / n) i, i=0,1,2, \ldots, n-1, \quad d_{i k}=2 r \sin \left[\left(\varphi_{i}-\varphi_{k}\right) / 2\right]$ $\varphi_{i k}=\left(\varphi_{i}+\varphi_{k}\right) / 2$ and $\mathrm{r}$ is the circle radius. Note that (14) is similar to a generic expression in (2) except for $\varphi_{i k}$, which, as we will see, plays a crucial role. In the case of small angular spread, $\Delta<<1$, the correlation between two adjacent elements can be approximated as in (3),

$$
R_{i(i+1)} \approx \operatorname{sinc}\left(\Delta d \cos \left(\varphi-\frac{\pi(2 i+1)}{n}\right)\right) e^{j 2 \pi d \sin \frac{\pi(2 i+1)}{n}}
$$

with $d=2 r \sin (\pi / n)$. Note that, in contrast to ULA, it depends on $i$, i.e. the pair location on the circle. In fact, $R_{i(i+1)}$ is periodic in $i$. Increase in d (i.e., the circle radius) will not eliminate the heavily correlated pairs due to the cos term in (15), which is in dramatic difference to the case of linear array. As it will be shown below, this has a profound effect on the capacity.

\section{MIMO CAPACITY AND DIVERSITY GAIN}

In this section, we analyze the impact of correlation on MIMO capacity and diversity gain. In particular, we demonstrate what is the effect of multiple clusters on these performance parameters.

\section{A. MIMO Capacity}

Using the approach of [2] and the correlation models above, the MIMO capacity can be evaluated. The channel is assumed to be quasi-static and frequency flat.

As an example, we consider a MIMO system of $n_{T}=n_{R}=10$ with $\mathrm{SNR}=20 \mathrm{~dB}$ per receive antenna, and a twocluster channel with 20 multipath signals arriving at each cluster (uniform pdf, with the same power per cluster); $\varphi_{1}=0^{0}$ (i.e., broadside), $\Delta_{2}=\Delta_{1}=10^{0}$ and the average AOA of the $2^{\text {nd }}$ cluster $\varphi_{2}=30^{\circ}, 60^{\circ}$ and $90^{\circ}$. The transmit array elements are assumed to be uncorrelated - all the correlation is concentrated at the Rx end. The mean capacity evaluated using the method of [2] is show in Fig. 6.

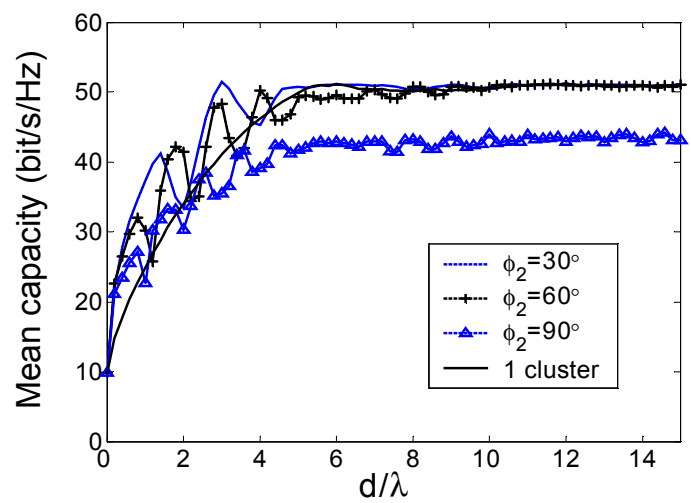

Fig. 6. 10x10 MIMO capacity of a two-cluster channel for various locations of the $2^{\text {nd }}$ cluster.

Clearly, moving the $2^{\text {nd }}$ cluster to the endfire direction results in lower capacity and lower capacity growth for small spacing. Hence, larger spacing is required to achieve the full capacity. This is in full agreement with the correlation behavior studied above. Also, the capacity oscillations, which do not appear in the single-cluster model [2], are apparent (this also follows from the correlation behavior analyzed above). Note that for $\varphi_{2}=90^{\circ}$ the capacity is smaller even for large $\mathrm{d}$ because of small contribution of the second cluster.

In the second example, we assume that the clusters are identical $\left(\Delta_{2}=\Delta_{1}=10^{0}\right)$ and orthogonal to each other, $\varphi_{1}=0^{0}, \varphi_{2}=90^{\circ}$, and we optimize the orientation of the $\mathrm{Rx}$ array (by rotation) to maximize the capacity. The scenario is depicted in Fig. 7.

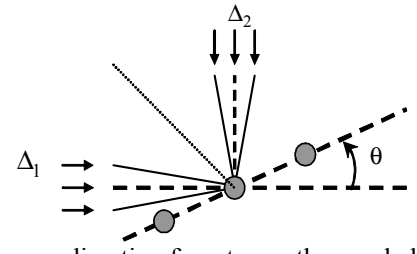

Fig. 7. Optimizing the array direction for a two orthogonal cluster channel 


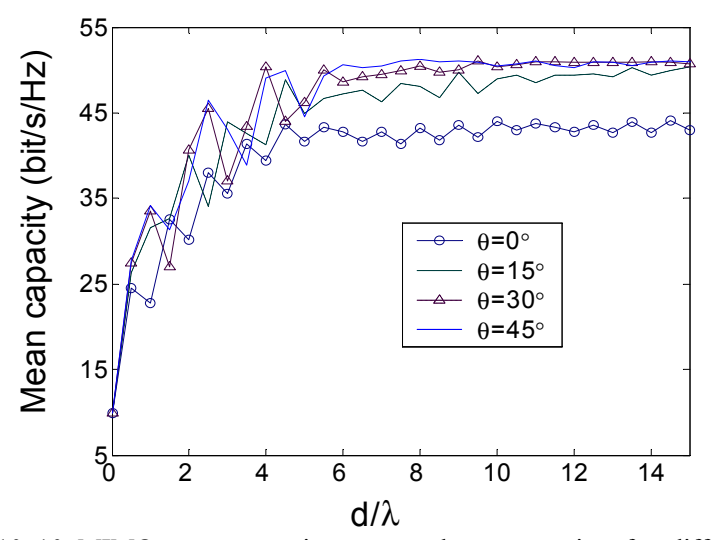

Fig. 8. 10x10 MIMO mean capacity versus element spacing for different orientations of the Rx array

Fig. 8 shows the mean capacity versus element spacing for different broadside array angles $\theta$. Clearly, the maximum capacity is achieved for most $\mathrm{d}$ when the array is oriented symmetrically with respect to the clusters, $\theta=45^{\circ}$ (i.e., the broadside direction coincides with the cluster symmetry line). Any deviation results in capacity decrease for most d. It is especially pronounced when the array endfire is located along one of the clusters, $\theta=0^{0}$, which basically eliminates the contribution of that cluster and results in lower capacity. Due to the geometry symmetry, the capacity behavior for $\theta \in\left[45^{0}, 90^{0}\right]$ is the same.

Finally, we compare capacities of the ULA and the UCA with the same aperture size and the number of elements. Detailed numerical analysis shows that the capacity of UCA is less than that of ULA for $d>10 \lambda$. We attribute this to high correlation of some element pairs on the UCA (as described in section IV). When the number of elements in the UCA is slightly increased, its capacity follows the same path as that of the ULA. Approximately $10 \%$ of the degrees of freedom are "lost" in the UCA due to the correlation.

\section{B. Diversity gain}

In the same way, we evaluate the diversity gain at a given outage probability using Monte-Carlo simulations.

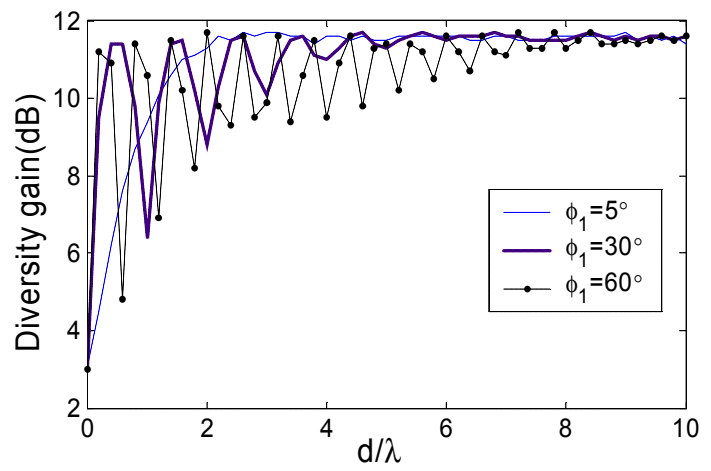

Fig. 9. Diversity gain of two-branch MRC at $P_{\text {out }}=10^{-2}$ for symmetric twocluster channel, $\Delta_{2}=\Delta_{1}=10^{0}$
As an example, we consider 2-branch MRC system with equal noise power per branch and evaluate its diversity gain at outage probability $P_{\text {out }}=10^{-2}$ for a symmetric two-cluster channel with $\Delta_{2}=\Delta_{1}=10^{0}$. The results are depicted in Fig. 9 . The case of $\varphi_{1}=5^{0}$ corresponds to a single-cluster channel (when two clusters merge into one). The maximum gain agrees well with the theoretical value of $11.5 \mathrm{~dB}$ (for a uncorrelated Rayleigh channel and $P_{\text {out }}=10^{-2}$ ). Note that the diversity gain oscillates as well, and the oscillation are more pronounced for larger $\varphi_{1}$. These oscillations are much larger than those of the capacity. Intuitively, this can be explained by an integral nature of the capacity (when all the local perturbations are averaged out). On the contrary, diversity gain is local in nature since it depends on rare evens (outage) and, hence, it is more sensitive to details of the angular pdf.

\section{CONCLUSIONS}

The analysis above demonstrates that the correlation in a twocluster channel can be significantly different from that of a single-cluster channel. The presence of the $2^{\text {nd }}$ cluster results in oscillations (with element spacing) of correlation and, hence, MIMO capacity and diversity gain. In some case, this can be beneficially used to decrease the element spacing while keeping the correlation low. The correlation depends significantly on the power distribution among the clusters. In some cases, the second cluster contribution is negligible. The reported results (in particular - correlation oscillations with spacing) agree well with recent measurements and provide a simple explanation of this phenomenon.

\section{REFERENCES}

[1] J. Salz, J.H. Winters, Effect of Fading Correlation on Adaptive Arrays in Digital Mobile Radio, IEEE Trans. Vehicular Technology, vol. 43, N. 4, pp. 1049-1057, Nov. 1994.

[2] S. Loyka, G. Tsoulos, Estimating MIMO System Performance Using the Correlation Matrix Approach, IEEE Communication Letters, v. 6, N. 1, pp. 19-21, Jan. 2002

[3] S. Loyka, A. Kouki, The Impact of Correlation on Multi-Antenna System Performance: Correlation Matrix Approach, 2001 IEEE Vehicular Technology Conference, Atlantic City, USA, Oct. 7-11, pp. 533-537.

[4] Jakes, W.C. Jr.: 'Microwave Mobile Communications', John Wiley and Sons, New York, 1974.

[5] D. Chizhik et al, Multiple-input-multiple-output measurements and modeling in Manhattan, IEEE Journal on Selected Areas in Communications, v. 21, N. 3, Apr 2003 , pp. 321 -331

[6] A. A. Saleh and R. A. Valenzuela, "A statistical model for indoor multipath propagation," IEEE J. Select. Areas Comm., vol. SAC-5, pp. 128-137, Feb. 1987

[7] Q. Spencer, M. Rice, B. Jeffs, and M. Jensen, "A statistical model for angle of arrival in indoor multipath propagation," in Proc. 1997 IEEE Veh. Tech. Conf., vol. 3, Phoenix, AZ, May 4-7, 1997, pp. 1415-1419

[8] K. I. Pedersen, P. E. Mogensen and B. H. Fleury, "A stochastic model of the temporal and azimuthal dispersion seen at the base station in outdoor propagation environments," IEEE Trans. Veh. Tech., Vol. 49, No.2, Mar. 2000, pp.437-447

[9] R.C. Johnson, Antenna Engineering Handbook, McGraw Hill, New York, 1993

[10] R. M. Buehrer, The Impact of Angular Energy Distribution on Spatial Correlation, IEEE Vehicular Technology Conference, Vancouver, Canada, September 24-28, 2002, pp.1173-1177

[11] L. Schumache et al, From Antenna Spacing to Theoretical Capacities - Guidelines for Simulating MIMO Systems, PIMRC 2002, pp. 587-592. 